Original Paper

\title{
Fully coupled FSI analysis of Francis turbines exposed to sediment erosion
}

\author{
Sailesh Chitrakar $^{1}$, Michel Cervantes ${ }^{2}$ and Biraj Singh Thapa ${ }^{3}$ \\ ${ }^{1}$ Department of Mechanical Engineering, School of Engineering, Kathmandu University \\ Turbine Testing Lab, Dhulikhel, Kavre, P.O. Box - 6250, Nepal, sailesh@ku.edu.np \\ ${ }^{2}$ Department of Fluid and Experimental Mechanics, Luleå University of Technology \\ 97187 Luleå, Sweden, michel.cervantes@1tu.se \\ ${ }^{3}$ Department of Mechanical Engineering, School of Engineering, Kathmandu University \\ Turbine Testing Lab, Dhulikhel, Kavre, P.O. Box - 6250, Nepal, bst@ku.edu.np
}

\begin{abstract}
Sediment erosion is one of the key challenges in hydraulic turbines from a design and maintenance perspective in Himalayas. The present study focuses on choosing the best design in terms of blade angle distribution of a Francis turbine runner which has least erosion effect without influencing the efficiency and the structural integrity. A fully coupled Fluid-Structure-Interaction (FSI) analysis was performed through a multi-field solver, which showed that the maximum stress induced in the optimized design for better sediment handling, is less than that induced in the reference design. Some numerical validation techniques have been shown for both CFD and FSI analysis.
\end{abstract}

Keywords: CFD, FSI, Francis, Sediment, Erosion

\section{Introduction}

The climatic and geographical scenarios of Nepal like tropical climate, immature geology and intense seasonal rainfall account for the degradation of the hydraulic turbine components from erosion and sedimentation. It has been reported that Southeast Asia alone contributes to two thirds of the world's total sediment transport to oceans which makes the problem of erosion and sedimentation even more challenging [1]. The vulnerability of the sediments is usually judged by the quartz content, as these materials have enough hardness to erode the turbine material. Even with well-designed sediment settling and flushing system, power plants have severe erosion problems. In Francis runner turbines, the most vulnerable regions to sediment erosion are 1) the inlet region due to high pressure difference between the pressure and the suction side of the runner and local erosion due to separation and 2) the outlet region, where the high relative velocity causes more erosion with the particles moving towards the outer diameter [1]. These localized effects are causing a non-uniform loss of the material and are difficult to repair. Hence, sediment erosion not only reduces efficiency in hydro turbines, but causes also various problems during the operation and maintenance periods.

The damage on hydraulic machineries due to sand erosion was initially studied in [2] by looking at various design aspects such as material selection, mechanics of material and hydraulics. The computational analysis of Francis turbines including the effect of erosion was done in [1], where the erosion rate was predicted for stay vanes, guide vanes and runner blades of a Francis turbine for different shape, size and concentration of the particle and operating conditions of the turbine. Numerical investigations carried out in the following works have shown that the conventional methods of hydraulic design of Francis turbines can be improved to minimize sediment erosion [3]. The comparison was done through computational fluid dynamics (CFD) approach by taking preimplemented erosion model in a commercial CFD code; Finnie and Tabakoff and Grant [3]. The modification of the blade angle distribution from inlet to outlet resulted in a significant change in the degree of erosion, without compromising much for the efficiency of the runner. The need of a structural analysis was felt when the material strength of the Francis runners was needed to be analyzed together with the hydraulic efficiency. However, the implementation of the Fluid-Structure-Interaction (FSI) has not been fully established for the case of Francis turbines, especially when exposed to sediment erosion. A one-way coupling strategy was presented in [4] to compare the structural integrity between the reference and the optimized designs. Such type of coupling is known to be insufficient to determine runner integrity because the flow field is assumed to be unaffected with the deflection of the runner, hence neglecting the two way effect. The importance of a two-way FSI technique has been highlighted and used for some

Received March 28 2014; revised May 22 2014; accepted for publication June 6 2014: Review conducted by Prof. Shuhong Liu. (Paper number O14007C)

Corresponding author: Sailesh Chitrakar, Research Fellow, sailesh@ku.edu.np 
other types of hydro turbines and pumps in [5] and [6]. Also, the mesh independence study, which is an integral part for the numerical validation, was seen to be difficult because of the extreme sensitiveness of the CFD results over the mesh density and distribution [7].

The present study addresses these computational challenges by performing a mesh sensitivity analysis for the selection of the optimized blade, and introducing a two way FSI for determining the structural integrity of Francis runners. All the simulations in this study were done considering a steady state assumption. However, the effect of the sediment erosion was taken into account in the structural analysis.

\section{Numerical models}

\subsection{CFD model}

The prediction of the sediment erosion on the blade was done based on Lagrangian calculation of particle paths in a viscous flow, by modeling a single passage of the runner in ANSYS CFX. The code uses a finite-volume multi-block approach to solve the governing equations of fluid motion numerically on a computational grid.

\section{Turbulence model}

Turbulence models are used to predict the effects of turbulence in fluid flow without resolving the small scale turbulent fluctuations. These models are based on RANS (Reynolds Averaged Navier-Stokes) equations. The most common eddy-viscosity models used are k- $\varepsilon, \mathrm{k}-\omega$ and SST models. For general purposes, the k- $\varepsilon$ model offers a good combination of accuracy and robustness. This model is, however, not suitable for predicting the boundary layer separation and flows in rotating fluids. In this study, the k- $\omega$ based Shear-Stress-Transport (SST) model was used as it provides a more accurate prediction of the flow separation under adverse pressure gradients [8].

\section{Erosion model}

The sediment was defined as a Quartz material with a density of $2.65 \mathrm{gm} / \mathrm{cm}^{3}$, Molar mass of $1 \mathrm{~kg} / \mathrm{kmol}$, diameter of $0.1 \mathrm{~mm}$ and spherical shape. The particle mass flow rate was chosen to be $1 \mathrm{~kg} / \mathrm{s}$ with uniform injection at a concentration of 1000 particles at the inlet. At present, ANSYS-CFX supports two erosion models. The first one is Finnie erosion model, in which the erosion is a function of the impact angle and velocity. In this study, the model of Tabakoff and Grant [9] was used for modeling the erosion on the blade with Quartz-Aluminum erosion parameters. In this model, the erosion rate E is determined from the following relation.

$$
E=k_{1} \cdot f(\gamma) \cdot V_{p}^{2} \cdot \cos ^{2}(\gamma)\left[1-R_{T}^{2}\right]+f\left(V_{P N}\right)
$$

In the above equation, $V_{P}$ is the particle impact velocity, $f(\gamma)$ is a dimensionless function of the impact angle in radian. The parameters $k_{1} R_{T}$ and $f\left(V_{P N}\right)$ are model constants depending on the particle/wall material combination and the impact velocity. It can be seen from this equation that the erosion rate predicted by Tabakoff and Grant is a highly non-linear function of impact velocity, impact angle and properties of eroding and eroded materials. Due to the dependency on more parameters than Finnie erosion model, Tabakoff and Grant erosion model is relatively more reliable. In this numerical study, particle transport was used as the multiphase model, where the particles carried by the fluid are tracked through Lagrangian approach, in which a set of differential equations for position, velocity and mass of the particle are integrated to calculate the behavior while travelling through the domain.

\section{Boundary conditions}

The main characteristics of the Jhimruk hydropower plant with design output of $12.6 \mathrm{MW}$ with 3 units, each running at $1000 \mathrm{rpm}$ were used in this study. The net head of the plant is $201.5 \mathrm{~m}$ and total discharge is $7.05 \mathrm{~m}^{3} / \mathrm{s}$. The curve files corresponding to the runner blade profile for generating the CFD domain were made from a Matlab based design program called 'Khoj', which was developed to create and optimize the design of Francis runners [10]. The same program was used to define the boundary parameters with the opening angle at best efficiency point.

\section{Erosion on the blade and particle tracking}

The study of the erosion on the blade can be done visually by looking at the erosion pattern on the blade and quantitatively by calculating the erosion rate density, which is defined as the mass of the eroded particle per unit surface area of the blade per unit time $\left(\mathrm{kg} \cdot \mathrm{m}^{-2} \mathrm{~s}^{-1}\right)$. Erosion can also be inferred from particle tracking, which shows the flow along the streamline of the quartz particle. Fig. 1 shows the reference blade with erosion pattern and the 1000 quartz particle tracks in the flow. It can be seen that the blade is mostly affected due to erosion towards outlet of the pressure side. It is because of the high relative velocity at the outlet of the runner that the particles moving towards the outer diameter in the runner will cause more erosion than towards the inlet. 


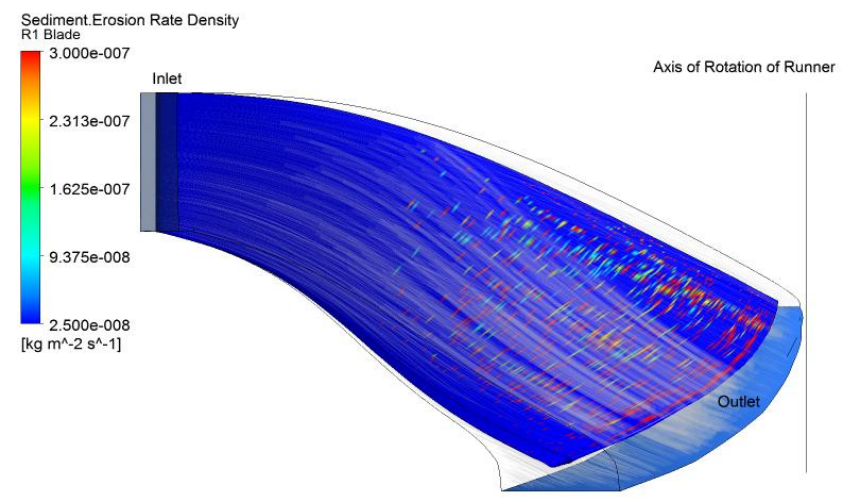

Fig. 1 Erosion pattern on the blade and tracking of the quartz particle in the flow

\section{Mesh study}

The CFD analysis for determining the sediment erosion rate density is extremely sensitive to the mesh density. Past studies show and support the difficulty in making the solution independent of the size of the mesh [7]. Although a y+ between 30 and 100 is recommended, to achieve such value requires fine mesh near the wall boundary, which needs a massive computational time. In Turbo-grid, the near wall mesh refinement allows a finer mesh around the blade wall, which can be controlled by changing the factor ratio i.e. the ratio of the size of the mesh between two successive layers. High value of the factor ratio increases the expansion rate and makes a finer mesh around the boundary, but it is also seen that it degrades the quality of the mesh. A factor ratio of 2 was used in the previous studies to create a very low y+ value as recommended in the studies near the walls. However, with this ratio, a very fine mesh was not possible because of the very low quality of the mesh. With such large ratio, some of the other parameters, such as the pressure field around the blade were affected. In this study, a factor ratio of 1.15 was chosen with the RMS value of $1 \mathrm{E}-6$ as residual criteria for convergence.

It is seen from Fig. 2 that for a mesh equal or larger than 0.75 million nodes, the average sediment erosion density becomes constant. This figure also shows the y+ values for various mesh nodes under study. For the most refined mesh, the average value of $\mathrm{y}+$ around the blade was about 37 .
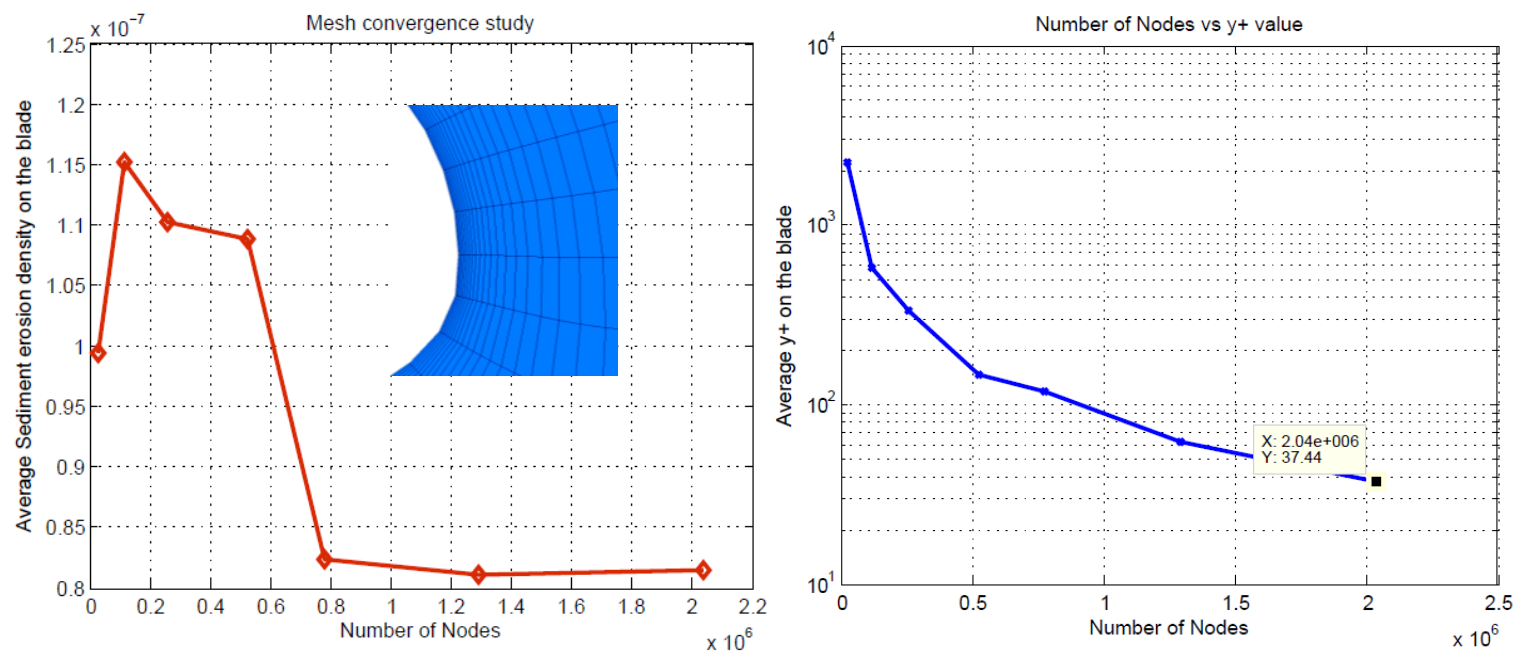

Fig. 2 Mesh convergence study for the factor ratio of 1.15, RMS of 1E-6 and y+ value on the blade

\section{Comparison between the optimized and the reference blades}

In the present study, various optimized blades are investigated, which were previously studied with CFD in [3]. The blades were modified based on the blade angle distribution from inlet to outlet. The graphical representation of these blades is shown in Fig. 3. In this figure, the blade angle represents the angle of the velocity with reference to the velocity of the runner in relative frame of reference. By changing the blade angle distribution, the relative velocity of the fluid changes from inlet to outlet. The shape 3 shows the linear distribution of the blade angle, which is chosen as the reference design. All the other designs and the results from the CFD are compared with this design. These blade shapes give an indication of how the hydraulic energy is converted to mechanical energy along the stream-wise direction. A runner blade design of shape 1 converts half of the hydraulic energy from the middle towards the outlet, whereas shape 2 will convert the energy at the beginning of the blade till the middle. 


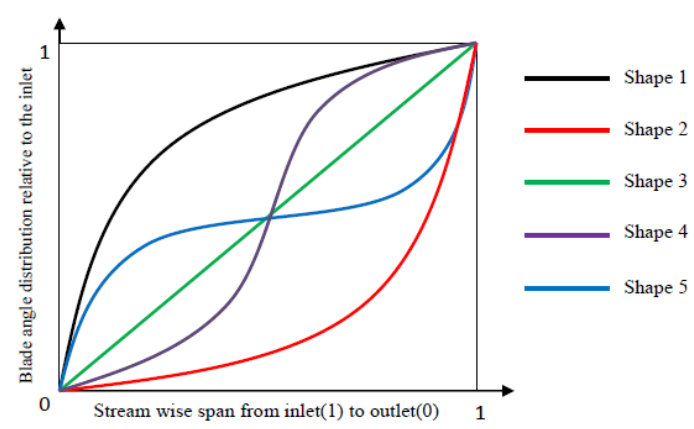

Fig. 3 Parametric study of the shape of the blades

The mesh independent model parameters were used to model all the optimized blades. Here, in order to study the sediment erosion pattern correctly, a mesh with 1.25 million nodes and a factor ratio of 1.15 were used for all the shapes with a RMS residual convergence target of 1E-6. In this case, two results are of interest: erosion and efficiency. The average erosion rate density on the blade with the erosion pattern and the efficiency are compared. The result of this analysis is shown in Fig. 4. In order to make it possible to plot the two variables in a single graph, the values are normalized with that of the reference design. This means that the reference shape (shape-3) has the value of 1 for both efficiency and erosion rate density, whereas the other shapes are relative to this shape. For convenience, their absolute values are also shown above the column, of which the erosion rate density is in the order of $1 \mathrm{E}-8$ and the unit of $\mathrm{kg} / \mathrm{m}^{2} \mathrm{~s}$. Also, the efficiency in percent is shown in the second column. The values on the abscissa represent the shape number of the blades under study.

It can be seen from this comparative analysis that the efficiency of the blades does not vary much with the change in the shape. However, the erosion rate density varies a lot. The blade shape 2 and 4 are the ones which show a decrease in the average erosion rate density on the blade compared to the reference shape. The decrease in this quantity is as much as $21 \%$ for the case of shape- 4 . Hence, the blade shape 4 is the most optimized blade in terms of erosion and efficiency and it is selected for the FSI analysis.

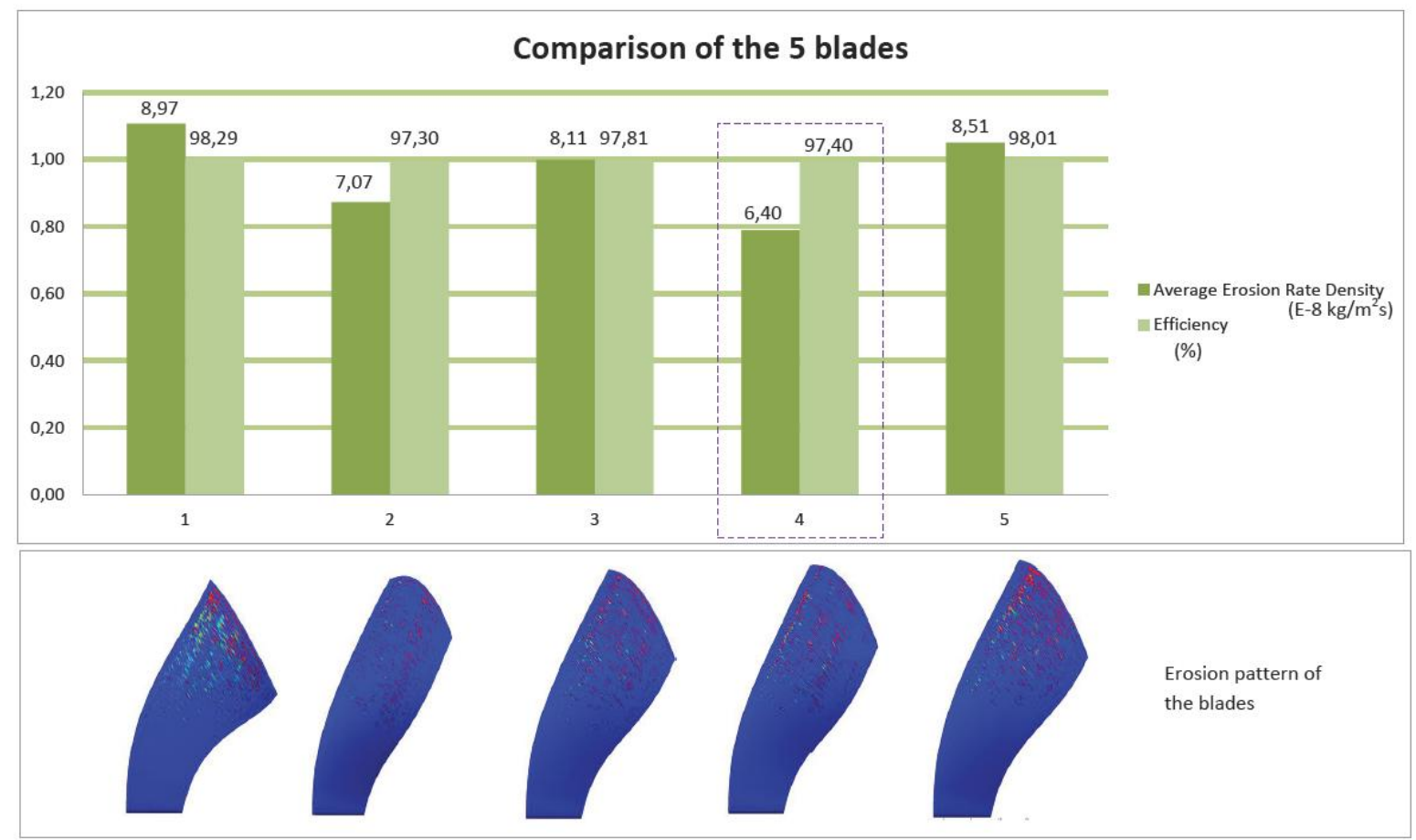

Fig. 4 Sediment erosion results for various shapes of the blade

\subsection{FSI model}

Fluid Structure Interaction (FSI) is a part of a coupled-field analysis where various independent fields combine and interact together to solve a global engineering problem with the result of one field dependent on the other. The coupling can either be oneway or two-way. In one-way coupling, the effect of one field is imposed on the other but not the vice versa. A two-way coupling is needed when the coupling between any two fields acquires significant response from each other. The coupling can be addressed by one of the two methods-direct or iterative. In the direct method, a single set of monolithic equation is assembled, leading to an increase in degrees of freedom of equations to be solved. Such methods require very high computational capability and hence, they are only used to solve simple structures. On the other hand, the iterative or load transfer approach assembles the equations for each field and field interfaces are used to transfer data.

This study focuses on MFX techniques, which uses an iterative approach for coupling from ANSYS to perform a two way FSI. 
MFX is an enhanced version of the multi-field solver used for simulations with physics fields i.e. fluid and structure distributed between more than one product executable (eg. Between ANSYS multiphysics or CFX and Mechanical). A field solver runs different codes involved in the coupled-interaction. These fields are then coupled using a form of iteration called 'stagger iteration'. The solution loop consists of two loops: the multi-field time loop and the multi-field stagger loop. The solution is divided into two different solvers, of which one is called master and another is called slave. The master performs the coupling setup (reads all the MFX commands, collects the interface meshes from the slave codes, does the mapping) and sends instructions (time and stagger loop controls) to the slave executable. Contrarily, the slave code receives the coupling information from the master code and sends the interface meshes to the master. In MFX, the ANSYS code is always the master, and CFX code is always the slave [11].

\section{Geometry}

The geometry of the blade, hub and shroud were made with the help of the curve files in Pro Engineer. The simplification of the problem was made by modeling a single section out of the 17 sections of the runner, containing a blade and proportional amount of hub and shroud. The boundaries of the structural domain should match with the fluid domain, so as to have a correct mapping between the two domains. The final geometry of the structural model is shown in Fig. 5.

Structural Steel was chosen as the structural material having following properties:

Density
Young's Modulus
Poisson's Ratio

Young's Modulus
Poisson's Ratio
$7850 \mathrm{~kg} / \mathrm{m}^{3}$

$2 \mathrm{E} 11 \mathrm{~Pa}$

0.3

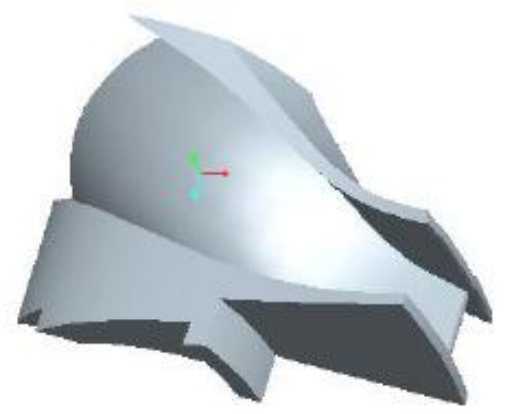

Fig. 5 Final geometry for structural analysis

\section{Boundary conditions}

Past research works on the Francis turbines have imposed various types of constraints for the structural analysis. In some cases, only the runner blade was considered without modeling the hub and the shroud and imposing fixed supports on the surface connecting them with the blade [12] [13]. Whereas, in some cases [14] [15], full geometry of the runner was considered, but the hub and the shroud were modeled with rigid supports, and their deformation was considered negligible with respect to the deformation of the blades. However, performing this analysis requires high computational cost and it is difficult to perform the mesh independent study for those models. Because of the symmetric property of the structure, it is more convenient to perform the analysis on a single sector in the same way as the CFD was done. The full analysis of the sector of the model by considering cyclic symmetry condition and deformation of the whole structure was done in [4]. In the current study, two types of boundary conditions were imposed, which are discussed below.

\section{Case I}

This case consists in a single blade with the following conditions:

- Zero displacement (fixed support) of the surface connecting blade-hub and blade-shroud.

- $\quad$ Rotational velocity about the $\mathrm{z}$-axis with $104.7 \mathrm{rad} / \mathrm{s}$.

- Acceleration due to gravity $(\mathrm{g})$.

- $\quad$ Fluid structure interface on the blade surface.

The reason behind conducting this analysis is to check the structural integrity of the blade without the influence of the other stiffer components. This will be easier when the comparison has to be made with the optimized blade. According to similar studies mentioned above, this type of analysis gives a reasonable estimation of the stress induced by the flow on the blade. However, the maximum stress could be less than the actual value because of the joint between the blade-hub and the blade-shroud. This analysis will be used as a starting solution to see the stress distribution and the deflection of the blade due to the pressure load from the flow field, without the influence of the other structural components and the neighboring blades.

\section{Case II}

This case consists of the following boundary conditions:

- Zero displacement (fixed support) of the surface connecting the shaft and the hub.

- $\quad$ Rotational velocity about the $\mathrm{z}$-axis with $104.7 \mathrm{rad} / \mathrm{s}$.

- Acceleration due to gravity (g).

- $\quad$ Fluid structure interface on the blade surface.

- $\quad$ Fluid structure interface on the hub and the shroud.

- Cyclic symmetry of the whole component. 


\section{FSI mesh study}

One way FSI simulations were used to perform the mesh study with the same boundary conditions as discussed above. The only difference is that instead of choosing the interface, the pressure load from CFD is directly imported into the boundary and the solution is obtained from Static Structural. Also, the cyclic symmetry property was defined by selecting the higher and the lower boundaries of the symmetric body. Doing this requires a new cylindrical co-ordinate system and the exact geometry on the higher and the lower side so the geometries and the mesh are properly mapped.

A mapped tetrahedron mesh was used to make the structural model of the blade and the unstructured mesh was used in the other regions. The mesh convergence study was performed on the first case only, and a relatively finer mesh was used to generate the results of the second case. The maximum equivalent stress and the maximum total deformation on the blades were studied for the convergence behavior. The result of this analysis for both the designs is shown in Fig. 6.

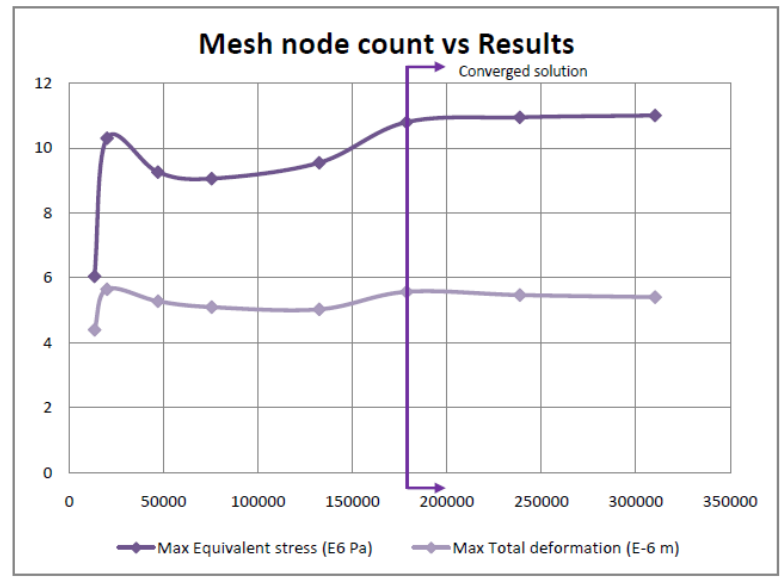

Reference

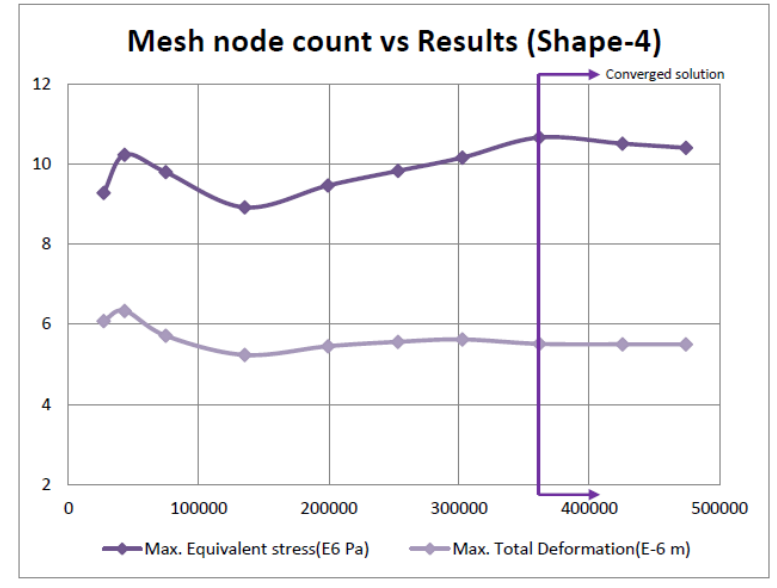

Optimized

Fig. 6 Mesh convergence study for structural analysis

For the reference design, after the mesh node count of 178575 , the solution is relatively converged. The change in the maximum stress is about $1 \%$ and that of the maximum total deformation is about $2 \%$, after this mesh size. Hence, this mesh node, corresponding to the element size of $0.0025 \mathrm{~m}$ was used to make the structural model of this design. On the other hand, for the optimized design, comparatively finer mesh density was needed to have the converged solution. The mesh node count of the mesh independent model was 361664 but the element size was only $0.0028 \mathrm{~m}$. For the second case, where the sector of the full runner was modeled, the element size of $0.004 \mathrm{~m}$ was used in both the designs. The reason for not conducting the mesh independence study for the second case, or for two way FSI was because of the limitations in the computational capability.

\section{Mesh deformation}

Mesh deformation is an important component for problems with moving boundaries or moving sub-domains. It is not considered when the steady CFD analysis was carried out. In the case of coupled FSI, this motion has to be imposed because the mesh outside the structural domain moves together with its deflection resulting in the deformation of the fluid mesh. This motion of the mesh could be determined by the mesh motion model. According to this model, the displacements applied on a boundary is diffused to mesh points with the equation,

$$
\nabla .\left(\tau_{\text {disp }} . \nabla \delta\right)=0
$$

Where, $\delta$ is the displacement relative to the previous mesh locations and $\tau_{d i s p}$ is the mesh stiffness. This equation is solved at the start of each outer iteration. It can be inferred from this equation that the relative mesh distribution of the initial mesh has been preserved. This means that the refinement of the mesh at the boundary will remain fine after the deformation at the relative position.

The value of the mesh stiffness discussed above can be controlled by choosing an appropriate value. This value can also be chosen such that the stiffness is more at the significant regions, such as near the small volumes, or near boundaries. The mesh stiffness value for this case is chosen according to the following relation,

$$
\tau_{\text {disp }}=\left(\frac{1}{a^{*}}\right)^{C_{s i f f}}
$$

Here $a^{*}$ represents either the size of the control mesh volumes, or the distance from the nearest boundary, depending upon the type of the option chosen. In any case, the stiffness of the mesh will increase when this value will decrease. The rate at which this stiffness increases depends upon the value of $C_{\text {stiff }}$ which represents the model exponent. The default value of this exponent is 10 , which can be altered according to the need of the problem.

In the case when ANSYS Multi-field is chosen for FSI, the mesh motion can be imposed on the wall boundaries. In this case, the mesh motion has to be imposed on the blade, hub and shroud regions. 


\section{Interface setup}

The load is transferred between the two domains at the interface. This is done through mapping of the nodes of one mesh to the local coordinates of an element in the other mesh. In the case of two ways coupling, two mappings are performed; first to map the displacements from solid nodes to fluid nodes and second to map the stresses from fluid to solid. ANSYS surfaces are flagged by interface numbers $(1,2 \ldots)$ and CFX surfaces are flagged by interface name (FSIN_1, FSIN_2...), these numbers and the name on the two domains should represent the same boundary.

The ANSYS Multi-field solver automatically transfers mesh-based quantities across dissimilar meshes. However, it is better to have similar size meshes between the two fields, to ensure the correct mapping between the fields. The quality of the mesh of the two fields is shown in Fig. 7. The distribution of the mesh is finer near the edges in the case of fluid, as this mesh was created from highly optimized topology option from Turbo-grid. Hence, the quality of the mesh is relatively better in the fluid domain.

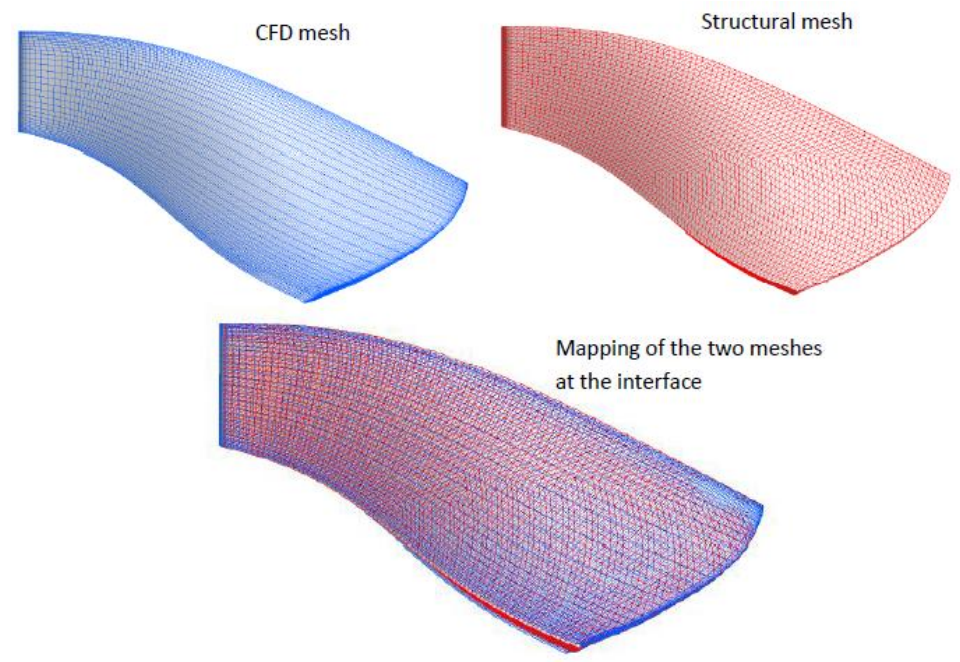

Fig. 7 Mesh of the two fields and mapping

\section{Solver setup}

In the case of the coupled-field analysis, the iteration controls and convergence criteria must be set for each field of the coupled field solvers. A special type of iteration, called stagger iteration is used to ensure the convergence of the quantities transferred between the two fields. At the end of each stagger iteration, the program as the master checks the convergence of the quantities transferred across the interface and the fields within each field solver. The stagger iteration continues unless the maximum number of stagger iteration is reached, or when the convergence has occurred. The convergence criteria for the stagger iteration can be chosen by defining the minimum value of a function $\phi$, given by the equation:

$$
\phi=\frac{\sqrt{\sum\left(u_{\text {new }}-u_{\text {old }}\right)^{2}}}{\sqrt{\sum\left(u_{\text {new }}\right)^{2}}}
$$

Where $u_{\text {old }}$ and $u_{\text {new }}$ are the load components transferred at the last and this stagger iteration respectively. The quantities are said to be converged when $\phi<\phi_{\min }$. By default, the value of $\phi_{\min }$ is set to 0.01 in ANSYS.

\section{Results of Two way FSI}

\section{Case-I}

The result of the two ways coupling for the first case, when only the blade was considered, is shown in Fig. 8. The maximum stress is towards the inlet region, at the shroud-blade connection. The value of the maximum stress is $12.45 \mathrm{MPa}$ for the reference design and 16.15 MPa for the optimized design. A large amount of stresses are induced on the zero displacement regions and also near the mid-span of the outlet region as shown in both the figure.

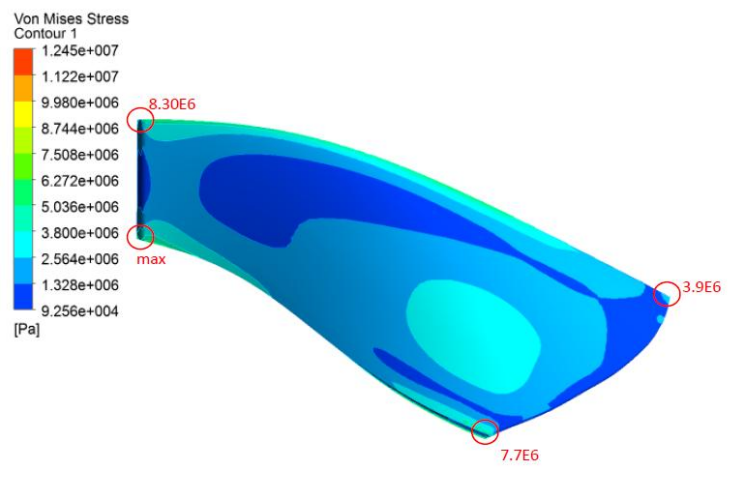

Reference

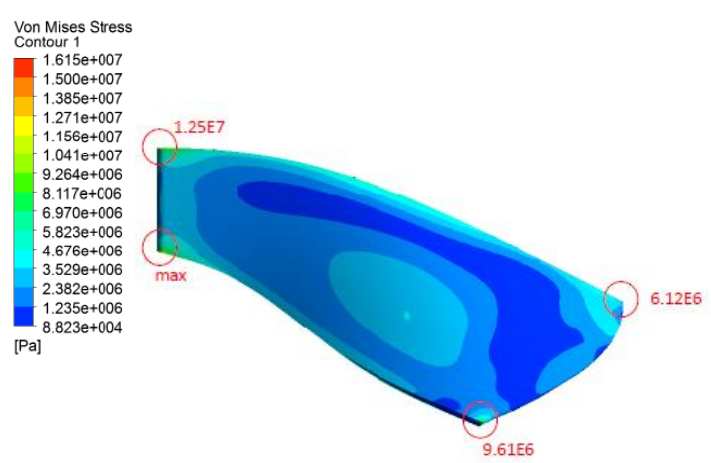

Optimized

Fig. 8 Stress distribution on the blade from two-way FSI 


\section{Case-II}

The stress distribution of the case-II for two way coupling is shown in Fig. 9. The region of the maximum stress is towards the blade-hub inlet region. A high stress distribution was found towards the joint between the blade-hub and the blade-shroud. The value of the maximum stress is $916.8 \mathrm{MPa}$ for the reference design and $823.9 \mathrm{MPa}$ for the optimized design.

In two way FSI, the deflection of the structure is represented by the deformation of the mesh of the flow field surrounding it. Fig. 10 shows the magnitude of the total mesh displacement around the blade at three different stream-wise positions. It also shows the overall deflection of the CFD mesh surrounding the blade. In the case of the reference design, the deformation of the mesh is maximum in the shroud region. This figure only shows the local displacement value on the legend. On the runner blade, the maximum deformation was found to be $0.37 \mathrm{~mm}$. In the case of the optimized design, the pattern of the deformation on the blade is quite different. Maximum deflection occurs towards the inlet and the midspan of the outlet region. Though the maximum deformation on the blade is less than the reference design $(0.12 \mathrm{~mm})$, the overall deformation of the CFD mesh is similar.

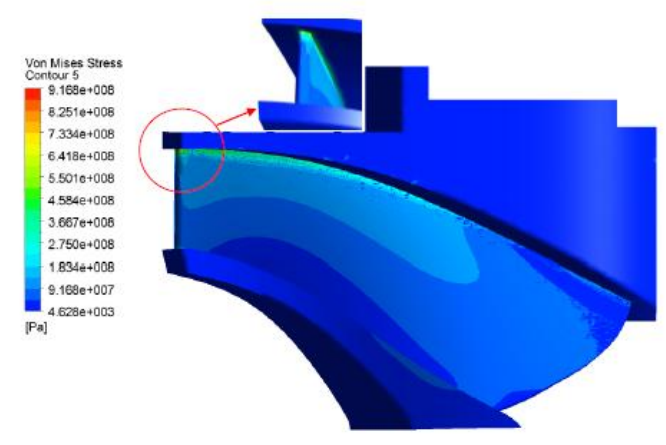

Reference

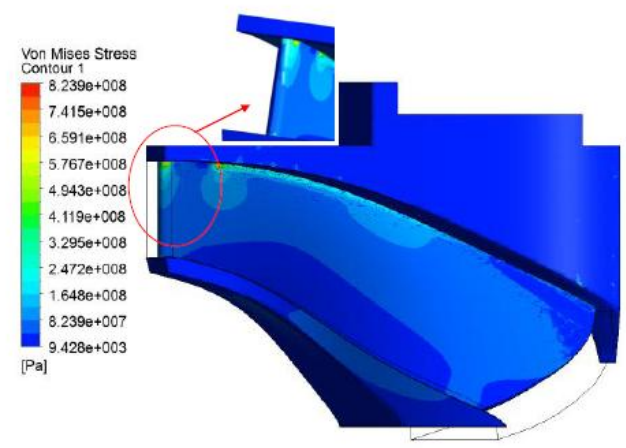

Optimized

Fig. 9 Stress distribution on the runner from two-way FSI
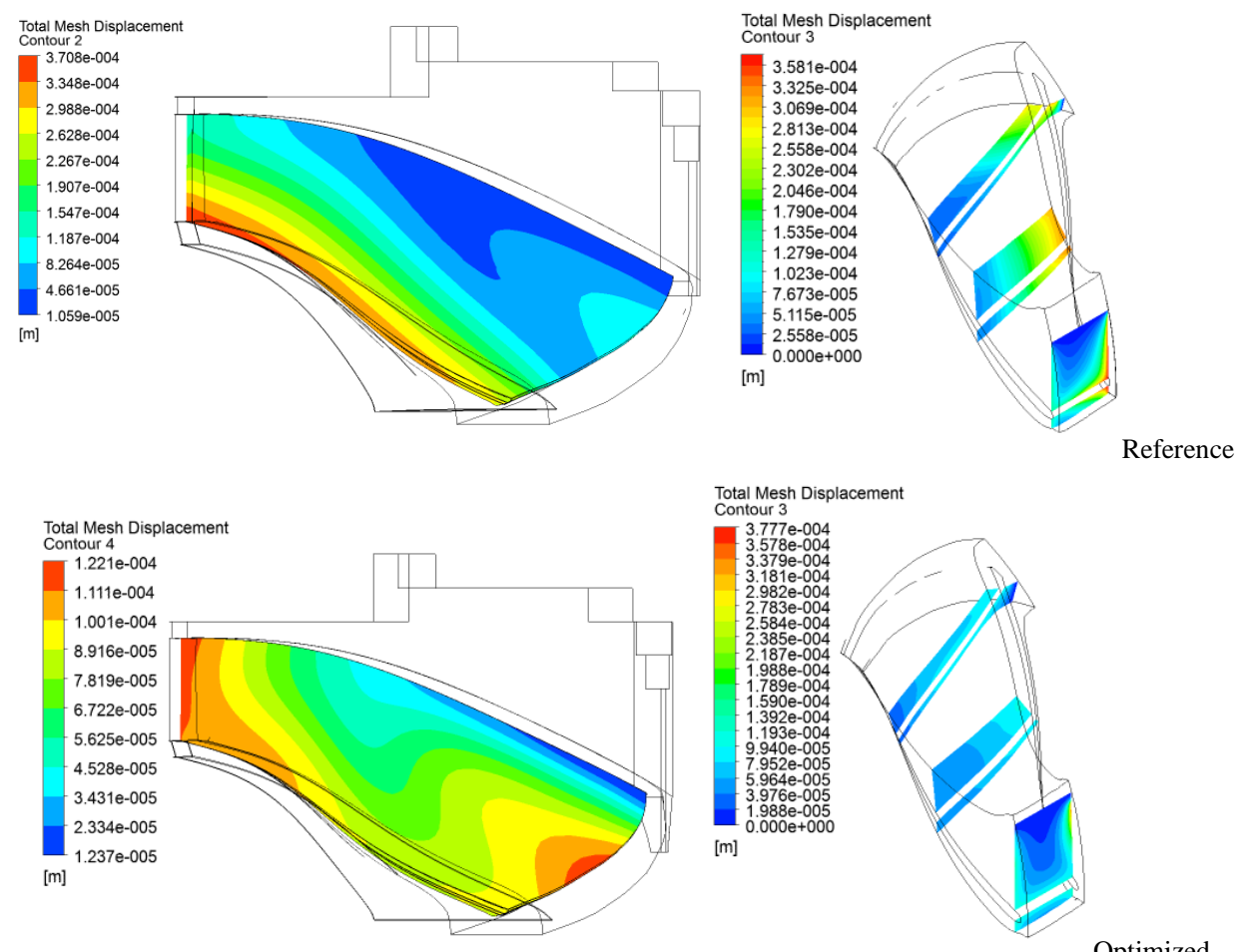

Optimized

Fig. 10 Mesh deformation in the fluid domain from two-way FSI

\section{Conclusion}

In this study, some of the computational techniques were used to identify the optimized design of a Francis runner in terms of sediment erosion, efficiency and structural integrity. CFD analysis was performed on a single blade passage, using the boundary conditions defined by a Matlab program developed in earlier studies, and using Tabakoff-erosion model supported by ANSYSCFX. Out of the 5 shapes compared, it was seen that shape-4 is the best design for minimizing erosion without changing 
efficiency and hence, it was used for FSI analysis. Two way FSI was performed on the runner section by taking two cases, first one taking a single blade without the influence of the joint and the neighboring blades, and second one taking a section of the full runner, by applying cyclic symmetry property. A mesh independent study was performed on the first case by considering a mapped tetrahedral mesh around the blade by implementing one way FSI. For the second case, a fixed size mesh was used in both the designs. It was seen that stress distribution on the runner in the second case was more than the first case, because of the inclusion of loadings on hub and shroud. The results of two way FSI showed that the maximum stress on the runner is less in the reference design compared to the optimized design in the first case, but it is opposite in the second case. The deflection of the runner could also be seen in the form of the deformation of the mesh around the structure, which shows that the deflection in the optimized blade is less than the deflection in the reference blade. In this paper, a complete technique for a multi-field problem has been presented by using a commercial software. The integrity of Francis turbines has been compared through three characteristics, erosion and efficiency using CFD and mechanical strength using a two-way FSI technique. In order to simplify the solution, the cyclic symmetry problem has been used to carry out the mesh independent study with reduced computational effort. The techniques used in this paper are equally applicable for related multi-disciplinary engineering applications. The findings from this study are also useful for developing a platform for a transient two-way coupling by considering the effect of stationary vanes.

\section{Acknowledgments}

Special thanks to Royal Institute of Technology, Sweden, Kathmandu University, Nepal and Luleå University of Technology, Sweden for their valuable contribution during the project duration.

\section{Nomenclature}

$\begin{array}{llll}\delta & \text { Displacement of the mesh } & \phi & \text { A function for convergence criteria } \\ \tau_{\text {disp }} & \text { Mesh stiffness } & \phi_{\text {min }} & \text { Convergence criteria } \\ C_{\text {stiff }} & \text { Model exponent } & u_{\text {old }} & \text { Previous load component } \\ a^{*} & \text { Size of mesh or distance from nearest boundary } & u_{n e w} & \text { Latter load component } \\ E & \text { Erosion rate density } & \gamma & \text { Impact angle }\end{array}$

\section{References}

[1] H. P. Neopane, 2010, "Sediment erosion in hydro turbines," NTNU.

[2] B. Thapa, R. Shrestha, P. Dhakal and B. S. Thapa, 2003, "Sediment in Nepalese hydropower projects," The Great Himalays: Climate, Health, Ecology, Management and Conservation.

[3] M. Eltvik, B. S. Thapa, O. G. Dahlhaug and K. Gjosaeter, 2012, "Numerical analysis of effect of design parameters and sediment erosion on a Francis runner," in Fourth International Conference on Water Resources and Renewable Energy Development in Asia, Thailand, 2012.

[4] J. B. Paulsen, 2012, "FSI-analysis of Francis turbine," NTNU, Norway.

[5] H. Schmucker, F. Flemming and S. Coulson, 2010, "Two-Way Coupled Fluid Structure Interaction Simulation of a Propeller Turbine," International Journal of Fluid Machinery and Systems, Vol. 3, No. 4, pp. 342-351.

[6] X. Huan, L. Houlin, T. Minggao, C. Jianbao, 2013, "Fluid-Structure Interaction Study on Diffuser Pump With a Two-Way Coupling Method" International Journal of Fluid Machinery and Systems, Vol. 6, No. 2, pp. 87-93.

[7] M. Eltvik, B.S. Thapa, O.G. Dahlhaug, K. Gjosaester, "Numerical analysis of effect of design parameters and sediment erosion on a Francis runner," International Journal of Hydropower and Dams, ASIA-2012.

[8] A. C. R. 11.0, “Ansys CFX-Solver Theory Guide,” 2006.

[9] ANSYS, Solver Theory Guide, Release 11.0, 2006.

[10] B. S. Thapa, M. Eltvik, K. Gjosaeter, O. G. Dahlhaug and B. Thapa, 2012, "Design optimization of Francis runners for sediment handling," in Water resources and Renewable energy Development in Asia, Thailand.

[11] A. R. 10.0, “ANSYS Coupled-Field Analysis Guide,” 2005.

[12] R. Negru, L. Marsavina and S. Muntean, 2011, “Analysis of Flow Induce Stress field in Francis turbine,” Bulletin of the Technical Insitute of Lasi.

[13] R. Negru, S. Muntean, L. Marsayina, R. Susan-Resiga and N. Pasca, 2011, "Computation of stress distribution in a Francis turbine runner induced by fluid flow," in International workshop on Computational Mechanics of Materials.

[14] R. A. Saeed, A. N. Galybin, V. Popov and N. O. Abdulrahim, 2009, "Modeling of the Francis turbine runner in power stations. Part II: Stress Analysis,” WIT transactions of the built environment, Vol. 105.

[15] S. Lais, Q. Henggeler, U. Weiss, E. X. and E. Eguasquiza, 2009, "Dynamic analysis of Francis runners-experiment and numerical simulation,” International Journal of Fluid Machinery and Systems, Vol. 2, No. 4, pp. 303-314. 